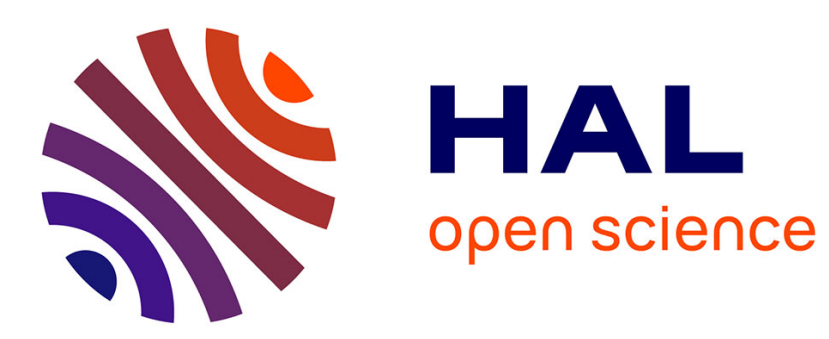

\title{
Une conduite cylindrique n'est pas optimale pour minimiser l'énergie dissipée par un fluide
}

\author{
Antoine Henrot, Yannick Privat
}

\section{To cite this version:}

Antoine Henrot, Yannick Privat. Une conduite cylindrique n'est pas optimale pour minimiser l'énergie dissipée par un fluide. Comptes Rendus. Mathématique, 2008, 346 (19-20), pp.1057-1061. 10.1016/j.crma.2008.09.005 . hal-00359518

\section{HAL Id: hal-00359518 https://hal.science/hal-00359518}

Submitted on 9 Feb 2009

HAL is a multi-disciplinary open access archive for the deposit and dissemination of scientific research documents, whether they are published or not. The documents may come from teaching and research institutions in France or abroad, or from public or private research centers.
L'archive ouverte pluridisciplinaire HAL, est destinée au dépôt et à la diffusion de documents scientifiques de niveau recherche, publiés ou non, émanant des établissements d'enseignement et de recherche français ou étrangers, des laboratoires publics ou privés. 


\title{
Une conduite cylindrique n'est pas optimale pour minimiser l'énergie dissipée par un fluide
}

\author{
Antoine Henrot ${ }^{\mathrm{a}}$, Yannick Privat ${ }^{\mathrm{a}}$ \\ ${ }^{a}$ Institut Élie Cartan Nancy, UMR 7502 Nancy-Université - CNRS - INRIA, B.P. 239, Vandøuvre-lès-Nancy Cedex \\ France
}

Reçu le 6 juin 2008; accepté après révision le 4 septembre 2008

Présenté par Pierre-Louis Lions

\begin{abstract}
Résumé
Nous considérons un écoulement de fluide incompressible dans un tuyau cylindrique tri-dimensionnel, régi par les équations de Navier-Stokes, avec des conditions aux limites classiques sur les parois du cylindre. Nous nous posons la question suivante : le cylindre est-il la forme optimale pour le critère "énergie dissipée par le fluide". Nous répondons par la négative, en explicitant la condition d'optimalité du premier ordre, à l'aide de l'état adjoint, et en montrant qu'il n'est pas possible que l'état adjoint soit solution du système surdéterminé ainsi obtenu.

Pour citer cet article: A. Henrot, Y. Privat, C. R. Acad. Sci. Paris, Ser. I (2008).
\end{abstract}

\section{Abstract}

A cylindrical pipe is not optimal to minimize the energy dissipated by a fluid We consider an incompressible fluid in a three-dimensional cylindrical pipe, following the Navier-Stokes system with classical boundary conditions on the boundary of the cylinder. We are interested in the following question: is the cylinder the optimal shape for the criterion "energy dissipated by the fluid"? We prove that it is not the case. For that purpose, we explicit the first order optimality condition, thanks to adjoint state and we prove that it is impossible that the adjoint state be a solution of this over-determined system.

To cite this article: A. Henrot, Y. Privat, C. R. Acad. Sci. Paris, Ser. I 340 (2008).

\section{Abridged English version}

The shape optimization problems in fluid mechanics are very important and gave rise to many works. Most often, these works have a numerical character due to the difficulty of the Navier-Stokes equations.

Email addresses: Antoine.Henrot@iecn.u-nancy.fr (Antoine Henrot), Yannick.Privat@iecn.u-nancy.fr (Yannick Privat). 
Nevertheless, for a result in the same spirit that ours, but in the context of (linear) Poiseuille flow, let us refer to [1] and [7]. For a first bibliography on the topic, we refer e.g. to [2], [4], [6], [8].

In this work, we are interested in one of the simplest problem: what shape must have a pipe in order to minimize the energy dissipated by the fluid? At first sight, intuition leads us to consider a cylindrical shape. We will show that it is not the optimal shape. Let us observe that Nature illustrates this result: in the lung, the bronchus are not cylindrical!

Let us consider a cylinder $\Omega \subset \mathbb{R}^{3}$ of equation $\left\{x_{1}^{2}+x_{2}^{2}<R^{2}, 0<x_{3}<L\right\}$ in which we have the flow of a viscous, incompressible fluid satisfying the Navier-Stokes system. We assume that the velocity profile $\mathbf{u}_{0}$ at the inlet $E=\left\{x_{3}=0\right\}$ is of parabolic type; on the lateral boundary $\Gamma$, we assume adherence condition $\mathbf{u}=0$ and we control the outlet by imposing an "outlet-pressure" condition on $S=\left\{x_{3}=L\right\}$. The p.d.e. system satisfied by the velocity $\mathbf{u}=\left(u_{1}, u_{2}, u_{3}\right)$ and the pressure $p$ is the Navier-Stokes system (1) where $\mathbf{h}=\left(h_{1}, h_{2}, h_{3}\right)$ and $\mathbf{u}_{0}$ are chosen in such a way that the flow follows a Poiseuille's law. This is not very realistic, but we need to know the exact solution in the case of a cylinder in order to write simply the optimality conditions. For a physical point of view, we must assume that the inlet and outlet pressure $p_{0}, p_{1}$ satisfy $p_{1}<p_{0}$. We will assume that the viscosity $\mu$ is large enough in order that the solution of (1) is unique (see [9]). More precisely, if we set $c=\frac{p_{1}-p_{0}}{4 \mu L}<0$, then the solution is given by

$$
\mathbf{u}\left(x_{1}, x_{2}, x_{3}\right)=\left(0,0, c\left(x_{1}^{2}+x_{2}^{2}-R^{2}\right)\right) \text { and } p\left(x_{1}, x_{2}, x_{3}\right)=4 \mu c\left(x_{3}-L\right)+p_{1} .
$$

The criterion we want to minimize is the energy dissipated by the fluid (or viscosity energy) defined by:

$$
J(\Omega):=2 \mu \int_{\Omega}|\varepsilon(\mathbf{u})|^{2} \mathrm{~d} x,
$$

where $\varepsilon$ is the stretching tensor :

$$
\varepsilon(\mathbf{u})=\left(\frac{1}{2}\left(\frac{\partial u_{i}}{\partial x_{j}}+\frac{\partial u_{j}}{\partial x_{i}}\right)\right)_{1 \leq i, j \leq 3}
$$

The aim of this note is to prove the following Theorem:

Theorem 0.1 Let us fix the inlet $E$ and the outlet $S$ (two identical parallel discs) of the domain $\Omega$ and let us consider a fluid satisfying to Navier-Stokes system (1).

The cylinder is not a solution of the shape optimization problem: $\min \left\{J(\Omega), \Omega \subset \mathbb{R}^{3}\right.$, meas $\left.(\Omega)=\pi R^{2} L\right\}$. The proof of this Theorem breaks down into different steps:

i Computation of the shape derivative of the criterion $J$. Using the classical tool of shape derivative (see e.g. [4]), we compute the derivative of the criterion $J$ when perturbations of the boundary $\Gamma$ occur. Introducing an adjoint state satisfying the system (6), we prove that the derivative of $J$, when $\Gamma$ is deformed with a vector field $\mathbf{V}$, is given by

$$
\mathrm{d} J(\Omega, \mathbf{V})=2 \mu \int_{\Gamma}\left(\varepsilon(\mathbf{u}): \varepsilon(\mathbf{v}) \mathrm{d} x-|\varepsilon(\mathbf{u})|^{2}\right)(\mathbf{V} . \mathbf{n}) \mathrm{d} \sigma .
$$

ii Optimality condition. Due to the volume constraint, we have a Lagrange multiplier relating derivative of the criterion and derivative of the volume. Finally, we are able to prove that the third component of the adjoint state $v_{3}$ must satisfy the overdetermined condition $\frac{\partial v_{3}}{\partial n}=0$ on the boundary $\Gamma$.

iii Mathematical analysis of the adjoint system. Studying carefully the adjoint system (6), we are able to prove some symmetry results due to the cylindrical symmetry of the domain.

iv Conclusion. We introduce a function $\psi\left(x_{3}\right)$ depending only on the last coordinate. Using the symmetry results and the optimality condition, we prove that this function is affine. Then, computing its 
(constant) derivative on the inlet and the outlet, we get $\psi^{\prime}(L)=-16 \mu c \pi R^{2}$ and $\psi^{\prime}(0)=-8 \mu c \pi R^{2}$. Therefore, $c=0$ (or $p_{0}=p_{1}$ ) a contradiction with the "physical" assumption.

\section{Introduction}

Les problèmes d'optimisation de forme en mécanique des fluides sont nombreux et importants (aile d'avion, turbines, pompes, ...) et ont déjà donné lieu à beaucoup d'études. Celles-ci sont souvent essentiellement numériques, car la difficulté intrinsèque des équations de Navier-Stokes rend difficile l'établissement de résultats théoriques. Néanmoins, nous renvoyons à [1] and [7] pour des résultats dans le même esprit que ceux que nous présentons ici, mais dans un cadre linéaire d'un écoulement de Poiseuille. Pour une première bibliographie sur le sujet, nous renvoyons par exemple à [2], [4], [6], [8] .

Dans ce travail, on considère l'un des problèmes les plus simples : quelle forme donner à une conduite pour minimiser l'énergie dissipée par le fluide. A première vue, l'intuition nous conduit à penser à une forme cylindrique. Nous allons montrer qu'il n'en est rien! Notons que la nature illustre ce résultat dans le poumon : nos bronches ne sont effectivement pas cylindriques.

Considérons donc un cylindre $\Omega \subset \mathbb{R}^{3}$ d'équation cartésienne : $\left\{x_{1}^{2}+x_{2}^{2}<R^{2}, 0<x_{3}<L\right\}$ dans lequel s'écoule un fluide visqueux incompressible régi par le système de Navier-Stokes. On se donne un profil de vitesses $\mathbf{u}_{0}$ parabolique à l'entrée $E=\left\{x_{3}=0\right\}$ du cylindre et on suppose la vitesse nulle sur la paroi latérale $\Gamma$ (condition d'adhérence). Sur la face de sortie $S=\left\{x_{3}=L\right\}$, on se donne une condition de type $\sigma(\mathbf{u}, p) \mathbf{n}=\mathbf{h}$ où $\sigma(\mathbf{u}, p)$ est le tenseur des contraintes. Il s'agit donc d'un écoulement contrôlé par la condition en sortie et on choisira $\mathbf{h}$ pour que la solution de l'écoulement dans un cylindre soit donné par la solution de Poiseuille. Notons que cette condition de sortie n'est pas très réaliste, car dans la pratique il n'y a pas de contrôle à la sortie du tuyau. On observe d'ailleurs pour ce type d'écoulement des tourbillons secondaires d'amplitude faible mais non nulle qui font qu'aucune des composantes de la vitesse ne s'annule (à cause de l'incompressibilité). La solution de Poiseuille qui vérifie $u_{1}=u_{2}=0$ n'est donc pas vraiment acceptable physiquement. Si nous l'avons choisie, c'est pour obtenir une expression plus simple des conditions d'optimalité (cf la 2ème étape de la preuve) qui nous permet une preuve complète.

Notons enfin $\mu>0$ le coefficient de viscosité cinématique. On supposera $\mu$ suffisamment grand pour que le système de Navier-Stokes ci-dessous possède une solution unique (cf [9]). Ainsi, le champ des vitesses $\mathbf{u}=\left(u_{1}, u_{2}, u_{3}\right)$, et la pression $p$ sont solutions du système de Navier-Stokes :

$$
\begin{cases}-\mu \triangle u_{i}+\frac{\partial p}{\partial x_{i}}+\sum_{j=1}^{3} \frac{\partial u_{i}}{\partial x_{j}} u_{j}=0 & \mathbf{x} \in \Omega, i \in\{1,2,3\} \\ \operatorname{div}(\mathbf{u})=0 & \mathbf{x} \in \Omega \\ \mathbf{u}=\mathbf{u}_{\mathbf{0}} & \mathbf{x} \in E \\ \mathbf{u}=0 & \mathbf{x} \in \Gamma \\ -p n_{i}+\mu \sum_{j=1}^{3}\left(\frac{\partial u_{i}}{\partial x_{j}}+\frac{\partial u_{j}}{\partial x_{i}}\right) n_{j}=h_{i} & \mathbf{x} \in S\end{cases}
$$

où $\mathbf{h}=\left(h_{1}, h_{2}, h_{3}\right)$ et $\mathbf{u}_{0}$ sont choisis de sorte que le fluide soit soumis à une loi de Poiseuille : $\mathbf{h}=$ $\left(\frac{p_{1}-p_{0}}{2 L} x_{1}, \frac{p_{1}-p_{0}}{2 L} x_{2},-p_{1}\right)$ et $\mathbf{u}_{0}=\left(0,0, \frac{p_{1}-p_{0}}{4 \mu L}\left(x_{1}^{2}+x_{2}^{2}-R^{2}\right)\right)$. 
Physiquement, il convient de choisir dans ce modèle $p_{1}<p_{0}$. Posons $c=\frac{p_{1}-p_{0}}{4 \mu L}<0$, alors, l'équation (1) possède une solution unique (voir par exemple [9]), donnée explicitement par :

$$
\mathbf{u}\left(x_{1}, x_{2}, x_{3}\right)=\left(0,0, c\left(x_{1}^{2}+x_{2}^{2}-R^{2}\right)\right) \quad \text { et } \quad p\left(x_{1}, x_{2}, x_{3}\right)=4 \mu c\left(x_{3}-L\right)+p_{1} .
$$

Le critère que nous cherchons à minimiser est l'énergie dissipée par le fluide (penser à une turbine ou un réacteur par exemple) ou énergie de viscosité définie par :

$$
J(\Omega):=2 \mu \int_{\Omega}|\varepsilon(\mathbf{u})|^{2} \mathrm{~d} x
$$

où $\varepsilon$ est le tenseur de déformations :

$$
\varepsilon(\mathbf{u})=\frac{1}{2}\left(\nabla \mathbf{u}+(\nabla \mathbf{u})^{T}\right)=\left(\frac{1}{2}\left(\frac{\partial u_{i}}{\partial x_{j}}+\frac{\partial u_{j}}{\partial x_{i}}\right)\right)_{1 \leq i, j \leq 3}
$$

L'objet de cette note est de démontrer le théorème suivant :

Théorème 1.1 On fixe l'entrée $E$ et la sortie $S$ (deux disques identiques parallèles) du domaine $\Omega$ et on considère un fluide satisfaisant aux équations de Navier-Stokes (1).

Le cylindre n'est pas solution du problème d'optimisation de forme:

$$
\left\{\begin{array}{l}
\min J(\Omega) \\
\operatorname{mes}(\Omega)=\pi R^{2} L .
\end{array}\right.
$$

\section{2. Étapes de la preuve}

Par manque de place, nous n'indiquons ci-dessous que les grandes étapes de la preuve. La preuve détaillée, ainsi que quelques compléments (système de Stokes, autre critère, résultats numériques) paraîtra dans [5].

1ère étape : Calcul de la dérivée de forme.

Le calcul de la dérivée par rapport au domaine (ou dérivée de forme) du critère $J$ défini par (3) est classique. Nous renvoyons par exemple à [4] pour la définition de cette dérivée et les détails. On obtient ici comme expression de cette dérivée où $V$ désigne un champ de déformation appliqué au bord latéral du domaine $\Omega$ (puisque $E$ et $S$ sont fixés, seul le bord latéral $\Gamma$ peut varier).

$$
\mathrm{d} J(\Omega, \mathbf{V})=2 \mu \int_{\Gamma}\left(\varepsilon(\mathbf{u}): \varepsilon(\mathbf{v}) \mathrm{d} x-|\varepsilon(\mathbf{u})|^{2}\right)(\mathbf{V} . \mathbf{n}) \mathrm{d} \sigma,
$$

où v est la solution du problème adjoint (6) suivant :

$$
\begin{cases}-\mu \triangle v_{i}+\sum_{j=1}^{3} v_{j} \frac{\partial u_{j}}{\partial x_{i}}-\sum_{j=1}^{3} u_{j} \frac{\partial v_{i}}{\partial x_{j}}+\frac{\partial q}{\partial x_{i}}=-2 \mu \triangle u_{i} & \mathbf{x} \in \Omega i \in\{1,2,3\} \\ \operatorname{div} \mathbf{v}=0 & \mathbf{x} \in \Omega \\ \mathbf{v}=\mathbf{0} & \mathbf{x} \in E \cup \Gamma \\ -q n_{i}+\mu \sum_{j=1}^{3}\left(\frac{\partial v_{i}}{\partial x_{j}}+\frac{\partial v_{j}}{\partial x_{i}}\right) n_{j}+\sum_{j=1}^{3}\left(u_{j} n_{j}\right) v_{i}-2 \mu \sum_{j=1}^{3}\left(\frac{\partial u_{i}}{\partial x_{j}}+\frac{\partial u_{j}}{\partial x_{i}}\right)=0 & \mathbf{x} \in S, i \in\{1,2,3\},\end{cases}
$$


2ème étape : Écriture de la condition d'optimalité.

En utilisant l'expression explicite de la solution donnée par (2), on a

$$
|\varepsilon(\mathbf{u})|^{2}=2 c^{2}\left(x_{1}^{2}+x_{2}^{2}\right) .
$$

Compte-tenu de la contrainte de volume, on a existence d'un multiplicateur de Lagrange $\lambda \in \mathbb{R}$, tel que

$$
\mathrm{d} J(\Omega, \mathbf{V})=\lambda \int_{\Gamma}(\mathbf{V} \cdot \mathbf{n}) \mathrm{d} s .
$$

En explicitant cette relation, on arrive finalement à l'existence d'un $\xi \in \mathbb{R}\left(\xi=\frac{\lambda-12 \mu c^{2} R^{2}}{2 \mu C R}\right)$ tel que, sur $\Gamma$, on a la condition "surdéterminée" :

$$
\frac{\partial v_{3}}{\partial n}=\xi
$$

3ème étape : Analyse mathématique du problème adjoint.

L'équation aux dérivées partielles (6) se réécrit, compte-tenu de l'expression explicite de $\mathbf{u}\left(x_{1}, x_{2}, x_{3}\right)$ :

$$
\begin{cases}-\mu \triangle v_{i}+2 c x_{i} v_{3}-\frac{\partial v_{i}}{\partial x_{3}}\left(x_{1}^{2}+x_{2}^{2}-R^{2}\right) c+\frac{\partial q}{\partial x_{i}}=0 & \mathbf{x} \in \Omega, i=1,2 \\ -\mu \triangle v_{3}-\frac{\partial v_{3}}{\partial x_{3}}\left(x_{1}^{2}+x_{2}^{2}-R^{2}\right) c+\frac{\partial q}{\partial x_{3}}=-8 \mu c & \mathbf{x} \in \Omega \\ \operatorname{div} \mathbf{v}=0 & \mathbf{x} \in \Omega \\ v_{1}=v_{2}=v_{3}=0 & \mathbf{x} \in E \cup \Gamma \\ \mu\left(\frac{\partial v_{i}}{\partial x_{3}}+\frac{\partial v_{3}}{\partial x_{i}}\right)+v_{i} c\left(x_{1}^{2}+x_{2}^{2}-R^{2}\right)=4 \mu c x_{i} & \mathbf{x} \in S, i=1,2 \\ 2 \mu \frac{\partial v_{3}}{\partial x_{3}}+v_{3} c\left(x_{1}^{2}+x_{2}^{2}-R^{2}\right)=q & \mathbf{x} \in S,\end{cases}
$$

On commence par montrer que les solutions du système (8) sont à "symétrie cylindrique". Cela demande un peu de travail : on utilise l'opérateur $\mathfrak{L}_{\theta}=x \frac{\partial}{\partial y}-y \frac{\partial}{\partial x}$ et, à plusieurs reprises, l'unicité de la solution d'un problème adjoint de type Stokes. Plus précisément, on obtient, en notant $r=\left(x_{1}^{2}+x_{2}^{2}\right)^{1 / 2}$ qu'il existe des fonctions $w, w_{3}$ et $\tilde{q}$ telles que, pour tout $\left(x_{1}, x_{2}, x_{3}\right) \in \Omega$ :

$$
\begin{aligned}
& \text { i } v_{i}\left(x_{1}, x_{2}, x_{3}\right)=x_{i} w\left(r, x_{3}\right), i \in\{1,2\}, \\
& \text { ii } v_{3}\left(x_{1}, x_{2}, x_{3}\right)=w_{3}\left(r, x_{3}\right), \\
& \text { iii } q\left(x_{1}, x_{2}, x_{3}\right)=\tilde{q}\left(r, x_{3}\right) .
\end{aligned}
$$

En utilisant cette symétrie, on montre à présent que dans la condition d'optimalité (7) on a nécessairement, $\xi=0$ et la condition d'optimalité se réécrit :

$$
\frac{\partial v_{3}}{\partial n}=0 \quad \text { sur } \Gamma .
$$

On en déduit également, en passant à la limite dans (8) que $q$ vérifie

$$
\frac{\partial q}{\partial n}=0 \quad \text { sur } \Gamma .
$$

4ème étape : Conclusion.

Introduisons, en utilisant les notations ci-dessus, les fonctions 


$$
w_{0}\left(r, x_{3}\right)=\int_{0}^{x_{3}} w(r, z) \mathrm{d} z \text { et } \psi(z)=\int_{T_{z}}\left(\tilde{q}-2 c r^{2} w_{0}\right) r \mathrm{~d} \theta \mathrm{d} r
$$

où $T_{z}$ désigne la section du cylindre $\left\{\left(x_{1}, x_{2}, x_{3}\right) \in \Omega / x_{3}=z\right\}$. Le point clé de la démonstration est le résultat suivant :

Lemme $2.1 \psi$ est une application affine.

Pour démontrer ce Lemme, on commence par prendre la divergence de l'équation satisfaite par l'état adjoint $(\mathbf{v}, q)$. Cela conduit à l'équation

$$
\triangle q+4 c v_{3}+2 c\left(x_{1} \frac{\partial v_{3}}{\partial x_{1}}+x_{2} \frac{\partial v_{3}}{\partial x_{2}}\right)-2 c\left(x_{1} \frac{\partial v_{1}}{\partial x_{3}}+x_{2} \frac{\partial v_{2}}{\partial x_{3}}\right)=0 .
$$

Ensuite, on intègre cette équation sur une tranche de cylindre de type $\left\{z_{-} \leq x_{3} \leq z_{+}\right\}$, puis on utilise plusieurs fois la formule de Green ainsi que (10) pour aboutir à $\psi^{\prime}(z)=a=$ constante.

Enfin, en travaillant sur la condition au bord satisfaite par $(\mathbf{v}, q)$ à la sortie du cylindre, puis à l'entrée, on obtient successivement $a=\psi^{\prime}(L)=-16 \mu c \pi R^{2}$ puis $a=\psi^{\prime}(0)=-8 \mu c \pi R^{2}$, d'où $c=0$ (ou $\left.p_{0}=p_{1}\right)$ une contradiction avec l'hypothèse "physique".

\section{Remerciements}

Nous remercions le referee anonyme pour ses suggestions pertinentes, en particulier sur la modélisation du problème.

\section{Références}

[1] G. Arumugam, O. Pironneau, On the problems of riblets as a drag reduction device, Optimal Control Appl. Methods 10 (1989), no. 2, 93-112.

[2] E. Feireisl, Shape optimization in viscous compressible fluids, Appl. Math. Optim., 47 (2003), no. 1, 59-78.

[3] G. P.Galdi An Introduction to the Mathematical Theory of the Navier-Stokes Equations Volumes 1 and 2, Springer Tracts in Natural Philosophy, Vol. 38, 1998

[4] A. Henrot, M. Pierre, Variation et Optimisation de formes, coll. Mathématiques et Applications, vol. 48, Springer 2005.

[5] A. Henrot, Y. Privat, The optimal shape of a pipe, to appear.

[6] B. Mohammadi, O. Pironneau, Applied shape optimization for fluids, Clarendon Press, Oxford 2001.

[7] O. Pironneau, G. Arumugam, On riblets in laminar flows, Control of boundaries and stabilization (Clermont-Ferrand, 1988), 53-65, Lecture Notes in Control and Inform. Sci., 125, Springer, Berlin, 1989.

[8] P. Plotnikov, J. Sokolowski, Shape optimization for Navier-Stokes equations, Control of coupled partial differential equations, 249-267, Internat. Ser. Numer. Math., 155, Birkhäuser, Basel, 2007.

[9] R. Temam Navier-Stokes Equations, North-Holland Pub. Company (1979), 500 pages. 